Journal Club

Editor's Note: These short, critical reviews of recent papers in the Journal, written exclusively by graduate students or postdoctoral fellows, are intended to summarize the important findings of the paper and provide additional insight and commentary. For more information on the format and purpose of the Journal Club, please see http://www.jneurosci.org/misc/ifa_features.shtml.

\title{
Decoding the Role of the Angular Gyrus in the Subjective Experience of Recollection
}

\author{
Alexandra Trelle \\ Department of Psychology, University of Cambridge, Cambridge CB2 3EB, United Kingdom \\ Review of Kuhl and Chun
}

Our knowledge of the neural mechanisms supporting episodic memory has developed dramatically since the 1950s through the use of neuropsychology research and, more recently, neuroimaging techniques. Although the roles of the medial temporal lobes and prefrontal cortex are well established, only recently has the involvement of the lateral parietal cortex (LPC) begun to receive attention. This interest arose from observations that the LPC, in particular the angular gyrus (ANG), is consistently active during the retrieval of episodic memories (Wagner et al., 2005; Vilberg and Rugg, 2008). This activation has been specifically linked to recollection, memory accompanied by contextual details from the original event, as opposed to familiarity, the feeling of knowing that a stimulus has been encountered previously, in the absence of retrieval of additional contextual details. For instance, using the classic remember/know paradigm, "remember" responses, which indicate that participants have experienced recollection, are associated with greater ANG activity compared with "know" responses, which signal feelings of familiarity (Vilberg and Rugg, 2008). Similarly, memory decisions about the context in which items were studied (source memory judgments), which rely on recollec-

Received Aug. 4, 2014; revised Sept. 3, 2014; accepted Sept. 8, 2014.

Correspondence should be addressed to Alexandra Trelle, Department

of Psychology, University of Cambridge, Downing Street, Cambridge CB2 3EB,UK. E-mail:ant24@cam.ac.uk.

DOI:10.1523/JNEUROSCI.3215-14.2014

Copyright $\odot 2014$ the authors $\quad 0270-6474 / 14 / 3414167-03 \$ 15.00 / 0$ tion, are associated with greater ANG activity than memory decisions about whether items were studied or not (item memory judgments), which can be supported by familiarity alone (Vilberg and Rugg, 2008).

In apparent contradiction to the neuroimaging evidence, patients with lesions to the LPC are not amnesic, typically achieving scores on many standard memory tests comparable to that of controls. Nonetheless, evidence suggests that such patients might exhibit subtle changes in recollection. These changes include declines in the proportion of "remember" responses relative to "know" responses in remember/know paradigms (Davidson et al., 2008), reduced confidence in source memory decisions despite intact accuracy (Simons et al., 2010), and a shift toward being less likely to judge with high confidence that an item was presented previously, regardless of the accuracy of their response (e.g., for both hits and false alarms; Hower et al., 2014). Together, these findings suggest that the LPC may support operations that contribute to the subjective experience of recollection, rather than being essential for the accurate retrieval of event details per se.

In many situations, objective recollection accuracy may be difficult to disentangle from one's subjective sense of successful recollection, as veridical recollection is typically accompanied by vivid episodic re-experiencing that results from the retrieval of contextual details from memory. However, when subjective mea- sures of recollection (e.g., confidence judgments or remember/know responses) are used to complement objective measures for which accuracy can be assessed (e.g., source memory judgments), studies in healthy individuals consistently observe a greater correspondence between ANG activity and the subjective experience of recollection. For instance, greater ANG activity is observed for high- relative to low-confidence responses (Yonelinas et al., 2005). Furthermore, "remember" judgments are associated with greater ANG activity than source memory judgments, and age-related declines in "remember" judgments have been linked to reductions in ANG activity (Duarte et al., 2008). Finally, there is evidence that increased ANG activity is associated with old responses, regardless of the item's true mnemonic history (e.g., whether it is in fact old or new), further bolstering the idea that ANG tracks one's subjective experience of recollection, or the "perceived oldness" of an event, rather than objective memory accuracy (Wheeler and Buckner, 2003).

Although the hypothesis that ANG is involved in the subjective experience of recollection appears to provide a parsimonious explanation for the results from neuroimaging and patient studies, the underlying mechanism by which ANG might support this vivid experience of recollection remains unclear. Several different, although not mutually exclusive, proposals have been put forward. Some of these suggest that ANG plays a more executive role 
in memory retrieval, accumulating mnemonic evidence in the service of informing memory decisions (Wagner et al., 2005) or directing attention to internal mnemonic representations (Cabeza et al., 2008), whereas others suggest that ANG actively supports the representation of retrieved content from memory, serving as an output buffer that maintains mnemonic details (Vilberg and Rugg, 2012) or potentially binding information from other cortical inputs (Shimamura, 2011). To evaluate these proposals, a recent study by Kuhl and Chun (2014) used multivoxel pattern analysis to characterize the neural representations supported by ANG during retrieval of previously studied wordpicture pairs. In particular, the authors were interested in determining the degree to which ANG (1) represents retrieved content from the study episode and (2) differentiates between individual memory events during retrieval.

Participants studied blocks of wordface and word-scene pairs before their memory was assessed with a cued recall task, wherein participants were presented with a word cue and instructed to silently recall the corresponding picture in as much detail as possible and provide a subjective indication of recall success by responding "vivid," "weak," or "don't know" if they were unable to recall the corresponding picture. The cued recall task was followed by an old/new recognition test for the images in the absence of the associated word pairing. This design effectively enabled the authors to address the two primary questions of interest. First, they could assess whether categoryspecific information (faces vs scenes) is represented in ANG during cued recall by testing how accurately these trials could be classified as face versus scene trials in the absence of pictorial input. Second, they were able to assess whether ANG supports event-specific representations by comparing activity associated with word cues during recall to that associated with pictures during recognition; test trials that share event information (e.g., a given word-picture pair) but, critically, lack perceptual overlap, thus ruling out perceptual processing explanations of the data. Finally, because a subjective measure of recollection success was used during the cued recall phase of the experiment, the design is also well suited to provide insight into the mechanisms through which ANG might contribute to the subjective experience of recollection.

The authors first conducted a wholebrain univariate analysis to identify brain regions associated with the subjective experience of vivid recollection. The contrast of recall trials associated with vivid remembering ("vivid" responses) to nonvivid recall trials ("weak"/don't know" responses) identified ANG among the regions that exhibited greater activity during vivid recollection, consistent with the subjective recollection hypothesis. All subsequent multivariate analyses were conducted with respect to 10 anatomical regions of interest (ROIs), including ANG, as well as a functional ROI ( $\left.\mathrm{ANG}_{\text {functional }}\right)$ that was created by selecting all voxels within the ANG anatomical mask that displayed greater activity in the subjective vividness contrast described above.

Using pattern classification analyses, robust reactivation of encoding-related category information (face or scene) was detected in ANG and $\mathrm{ANG}_{\text {functional }}$ during cued recall trials, indicating that voxels associated with the subjective experience of vivid remembering also carried information about the category of the recalled picture in the absence of pictorial input. Furthermore, $\mathrm{ANG}$ and $\mathrm{ANG}_{\text {functional }}$ ROIs were the only regions identified to successfully differentiate between individual retrieval events. In these regions, pattern similarity analyses revealed greater overlap between recall and recognition trials corresponding to the same wordpicture pair, relative to noncorresponding trials belonging to the same image category. Interestingly, the category and event information detected in ANG was only reliable for trials in which participants indicated that recall was successful (e.g., received a "vivid" response), suggesting that these representations are not simply associated with a general retrieval attempt, but are specific to successful recollection, as judged by the participant.

Together, these results favor an account of the mechanism by which ANG supports subjective aspects of recollection that involves the active representation of event content retrieved from memory. Both the output buffer proposal and the binding proposal are consistent with this idea, suggesting that ANG might support the temporary maintenance of details associated with the study episode, possibly binding the various constituents into a coherent event representation. Although the specific nature of the event-specific representations supported by ANG remains unclear (e.g., whether these comprise perceptual or semantic content, other phenomenological aspects of the retrieval event, or some combination of these elements), this information was only present on trials that participants deemed successful, which is consistent with the proposal that these representations actively contribute to subjective aspects of recollection. Thus, these results provide evidence for a mechanism that can explain how ANG might support the subjective experience that accompanies remembering that is compatible with findings observed in both patient and neuroimaging studies.

One issue with the present study is that recall trials did not include an objective measure of recollection; instead the analyses relied exclusively on participants' subjective reports of recall success. As a result, veridical recall accuracy remains unclear. Although category information could be decoded from neural activity during successful recall trials, and this suggests that participants successfully recalled whether the presented cue word was associated with a face or a scene, the degree to which additional details of the study event were retrieved with objective accuracy, including specific conceptual or perceptual details associated with the original image, cannot be ascertained. Accordingly, one should be cautious in interpreting the degree to which the evidence presented here extends beyond subjective recollection success. Furthermore, although these results are consistent with the subjective recollection hypothesis of ANG function, they cannot determine whether the information carried by ANG during successful recollection is a function of objective memory accuracy, or related to the subjective experience that so often accompanies veridical recollection.

To test the predictions of the subjective recollection hypothesis, namely that the computations supported by ANG make particular contributions to the subjective experience of recollection which, depending on the scenario, may provide a basis for objective recollection accuracy decisions, it will be critical for future studies to include both subjective and objective measures of recollection. For example, cued recall could be accompanied by subjective vividness or confidence judgments as well as a specific question regarding perceptual or conceptual details of the recalled stimulus. Alternatively, more extensive memory tests for stimulus details could also be administered postscan. A significant benefit of such a design would be the ability to contrast the nature of ANG representations during high-confidence-correct trials with those of high-confidence-incorrect trials and lowconfidence-correct trials. The subjective experience of recollection hypothesis 
would predict that the presence of retrieved content and event-specific information in ANG would be associated with subjective recollection success, even in the absence of objective memory accuracy; likewise, this information should be less reliable on trials in which objective accuracy is not accompanied by a vivid experience of recollection.

Although the present research neither confirms nor contradicts these predictions, the results point to a possible mechanism by which ANG enables the subjective experience of recollection, namely through the active representation of retrieved content and event-specific information. A critical objective of future research is to further specify the nature of these representations. The use of more complex multimodal stimuli such as short film clips, or the retrieval of autobiographical memories may be useful in this regard, as these are likely to placer greater demands on maintenance and integration processes that may be supported by ANG during retrieval. Accordingly, such an approach may enable us to further adjudicate between alternative accounts of how ANG supports the rich and vivid re-experiencing of episodic memories.

\section{References}

Cabeza R, Ciaramelli E, Olson IR, Moscovitch M (2008) The parietal cortex and episodic memory: an attentional account. Nat Rev Neurosci 9:613-625. CrossRef Medline

Davidson PS, Anaki D, Ciaramelli E, Cohn M, Kim AS, Murphy KJ, Troyer AK, Moscovitch M, Levine B (2008) Does lateral parietal cortex support episodic memory? Evidence from focal lesion patients. Neuropsychologia 46: 1743-1755. CrossRef Medline

Duarte A, Henson RN, Graham KS (2008) The effects of aging on the neural correlates of subjective and objective recollection. Cereb Cortex 18:2169-2180. CrossRef Medline

Hower KH, Wixted J, Berryhill ME, Olson IR (2014) Impaired perception of mnemonic oldness, but not mnemonic newness, after parietal lobe damage. Neuropsychologia 56: 409-417. CrossRef Medline

Kuhl BA, Chun MM (2014) Successful remembering elicits event-specific activity patterns in lateral parietal cortex. J Neurosci 34:80518060. CrossRef Medline

Shimamura AP (2011) Episodic retrieval and the cortical binding of relational activity. Cogn Affect Behav Neurosci 11:77-291. CrossRef Medline

Simons JS, Peers PV, Mazuz YS, Berryhill ME, Olson IR (2010) Dissociation between memory accuracy and memory confidence following bilateral parietal lesions. Cereb Cortex 20:479-485. CrossRef Medline

Vilberg KL, Rugg MD (2008) Memory retrieval and the parietal cortex: a review of evidence from a dual-process perspective. Neuropsychologia 46:1787-1799. CrossRef Medline

Vilberg KL, Rugg MD (2012) The neural correlates of recollection: transient versus sustained FMRI effects. J Neurosci 32:15679-15687. CrossRef Medline

Wagner AD, Shannon BJ, Kahn I, Buckner RL (2005) Parietal lobe contributions to episodic memory retrieval. Trends Cogn Sci 9:445453. CrossRef Medline

Wheeler ME, Buckner RL (2003) Functional dissociation among components of remembering: control, perceived oldness, and content. J Neurosci 23:3869-3880. Medline

Yonelinas AP, Otten LJ, Shaw KN, Rugg MD (2005) Separating the brain regions involved in recollection and familiarity in recognition memory. J Neurosci 25:3002-3008. CrossRef Medline 